\title{
Automated screening of blood cultures with the Malthus microbiological growth analyser
}

\author{
D F J BROWN, M WARNER, C E D TAYLOR, R E WARREN Clinical Microbiology and \\ Public Health Laboratory, Addenbrooke's Hospital, Cambridge
}

SUMMARY A total of 3347 blood cultures from patients in all hospital wards were examined on a Malthus microbiological growth analyser and by a conventional system. There was no significant difference in the total numbers of positive cultures of clinical importance between the two systems ( $p$ $>0.05$ ). Staphylococcus aureus, however, was isolated more often by the conventional method ( $\mathrm{p}<$ 0.05). Failure of the automatic detection routine limited the potential of the Malthus system for earlier detection of positive cultures. Daily visual examination of Malthus curves and subculture of bottles not promptly attached to the apparatus were necessary to avoid missing some positive cultures. False positive rates were $13 \%$ for the Malthus system and $2 \%$ for the conventional system. The contamination rate was considerably lower in the Malthus system $(\mathrm{p}<0.001)$.

Further development would be necessary for the apparatus to be acceptable for routine screening of blood cultures.

A preliminary trial of a prototype Malthus microbiological growth analyser suggested that the apparatus might provide results more quickly than a conventional system of blood culture.' A fully automated system might also eliminate time consuming and subjective visual examination of bottles and blind subcultures - costly in terms of labour and materials, and which carries the risk of contamination. The prototype Malthus system, however, required manual interpretation of data presented graphically on a visual display unit. Consequently the potential benefits of automation were not realised. In the current trial the Malthus system automatically examined the data and signalled on the visual display unit when the programmed detection routine was triggered.

The previous trial ${ }^{1}$ was conducted in a research laboratory with specimens almost exclusively from patients with haematological malignant disease, receiving haemodialysis or recovering from renal transplantation. Hence, results may not have been typical of those obtained routinely with specimens from patients with various conditions. In the current study the apparatus was run by staff in the routine laboratory, specimens being obtained from all wards in the hospital.

Accepted for publication 30 January 1988

\section{Material and methods}

\section{BLOOD CULTURES}

Clinicians were requested to take $30 \mathrm{ml}$ blood and to distribute it equally between two bottles each containing $85 \mathrm{ml}$ medium. Blood culture media used were as previously described.' One bottle was a Malthus cell (Malthus Instruments Ltd) and the other a $120 \mathrm{ml}$ medical flat bottle. Forty five millilitres of blood were taken from patients with haematological malignant disease and an additional conventional bottle inoculated. The medium, however, differed from that in the other bottles. Results from the additional bottle were therefore excluded. Results of samples from children are also excluded because the volume of culture medium differed between conventional and Malthus bottles and blood samples were not equally distributed between the bottles in each pair.

\section{CONVENTIONAL BLOOD CULTURES}

These were processed as previously described, ${ }^{1}$ except that bottles were examined daily for turbidity for nine days and when turbidity of the medium suggested bacterial growth, bottles were subcultured by means of a Pasteur pipette. Organisms isolated were identified by methods described elsewhere ${ }^{2}$ and by the use of API kits for non-fermenting Gram negative bacilli, streptococci, and yeasts (API Laboratory Products). 
MALTHUS BLOOD CULTURES

These were processed essentially as previously described. ' The Malthus microbiological growth analyser (Malthus Instruments Ltd) was a production model of that used earlier. Conductivity recordings were also visually examined daily to detect positive cultures if the automatic detection routine had failed. For a period of three months during the trial, the value of examining cultures which gave a positive signal during the evening was assessed by use of an automatic dialling telephone to interrogate the apparatus at $22.00 \mathrm{~h}$. In the event of a positive message a member of staff returned to the laboratory to subculture appropriate bottles.

The significance of differences in isolation rates was assessed by the use of McNemar's test with a correction for continuity. ${ }^{3}$

\section{Results}

\section{POSITIVE CULTURES}

Of the 3347 paired cultures, organisms considered to

Table 1 No of significant isolates from various bottles be clinically important were isolated from both bottles in 213 cultures, from the Malthus bottle alone in 57 cultures, and from the conventional bottle alone in $\mathbf{6 0}$ cultures. The difference in isolation rates between the bottles was not significant ( $p>0.05$ ).

Isolates assessed clinically to be contaminants were principally Staphylococcus epidermidis but also included Bacillus spp, non-fermenting Gram negative rods, streptococci, coryneform organisms and Aspergillus spp. Contaminants were isolated from both bottles in 64 cultures, from the Malthus bottle alone in 50 cultures, and from the conventional bottle alone in 387 cultures. Differences in contamination rates were highly significant $(\mathrm{p}<0.001)$.

\section{ORGANISMS ISOLATED}

There were no significant differences in the total numbers of isolates from the conventional (287 isolates) and Malthus (282 isolates) bottles (table 1). There were, however, significantly more isolates of $S$ aureus from the conventional bottle than from the Malthus bottle $(\mathrm{p}<0.05)$.

\begin{tabular}{|c|c|c|c|}
\hline Organisms isolated & Both bottles & Conventional alone & Malthus alone \\
\hline Escherichia coli & 53 & 4 & 10 \\
\hline Klebsiella spp & 16 & 4 & 6 \\
\hline Enterobacter cloacae & 4 & 1 & 2 \\
\hline Enterobacter agglomerans & 0 & 0 & 1 \\
\hline Enterobacter sp & 1 & 1 & 0 \\
\hline Proteus mirabilis & 4 & 0 & 4 \\
\hline Proteus vulgaris & 2 & 0 & 0 \\
\hline Morganella morganii & $\overrightarrow{1}$ & 0 & 1 \\
\hline Serratia liquefaciens & 2 & 0 & $i$ \\
\hline Serratia spp & 1 & 1 & 2 \\
\hline Salmonella typhi & 2 & 1 & 1 \\
\hline Citrobacter freundii & 1 & 0 & 0 \\
\hline Acinetobacter spp & 0 & 2 & 0 \\
\hline Alcaligens spp & 0 & 2 & 0 \\
\hline Aeromonas spp & 2 & 0 & 1 \\
\hline Pseudomonas maltophilia & 0 & 3 & 1 \\
\hline Pseudomonas spp & 10 & 3 & 3 \\
\hline Haemophilus influenzae & 5 & 1 & 0 \\
\hline Staphylococcus aureus & 28 & 14 & 4 \\
\hline Staphylococcus epidermidis & 25 & 9 & 6 \\
\hline Group A streptococcus & 3 & 0 & 0 \\
\hline Group B streptococcus & 2 & 1 & 0 \\
\hline Group G streptococcus & 0 & 1 & 1 \\
\hline Streptococcus faecalis & 11 & 1 & 1 \\
\hline Streptococcus faecium & 10 & 5 & 3 \\
\hline Streptococcus bovis II & 1 & 0 & 0 \\
\hline Streptococcus sanguis I & 1 & 0 & 0 \\
\hline Streptococcus sanguis II & 0 & 2 & 0 \\
\hline Streptococcus mitis & 1 & 1 & 0 \\
\hline Streptococcus milleri II & 3 & 1 & 1 \\
\hline Streptococcus pneumoniae & 23 & 2 & 4 \\
\hline Coryneform & 1 & 0 & 0 \\
\hline Capnocytophaga ochracea & 0 & 0 & 1 \\
\hline Clostridium perfringens & 1 & 1 & 4 \\
\hline Clostridium butyricum & 1 & 0 & 1 \\
\hline Clostridium tertium & 0 & 0 & 2 \\
\hline Bacteroides melaninogenicus & 1 & 0 & 0 \\
\hline Bacteroides capillosus & $i$ & 0 & 0 \\
\hline Bacteroides spp & $i$ & 2 & 3 \\
\hline Peptococcus sp & 0 & $\overrightarrow{1}$ & 0 \\
\hline Candida albicans & 0 & 1 & 0 \\
\hline Candida tropicalis & 0 & $i$ & 0 \\
\hline Torulopsis glabrata & 0 & 3 & 0 \\
\hline Total No of significant isolates & 218 & 69 & 64 \\
\hline
\end{tabular}


Table 2 Organisms not detected by Malthus automatic detection routine, or conventional system before terminal subculture

\begin{tabular}{|c|c|c|c|c|}
\hline & \multicolumn{2}{|c|}{ Significant cultures } & \multicolumn{2}{|c|}{ Contaminated cultures } \\
\hline & Malthus & Conventional & Malthus & Conventional \\
\hline Streptococcus pneumoniae & 14 & 1 & 0 & 0 \\
\hline Streptococcus mitis & 1 & 0 & 0 & 0 \\
\hline Streptococcus milleri & 2 & 0 & 0 & 0 \\
\hline Streptococcus bovis & 1 & 0 & 0 & 0 \\
\hline Streptococcus salivarius & 0 & 0 & 1 & 0 \\
\hline Streptococcus sanguis & 0 & 0 & 3 & 0 \\
\hline Streptococcus faecalis & 1 & 0 & 0 & 0 \\
\hline Staphylococcus aureus & 8 & 1 & 0 & 0 \\
\hline Staphylococcus epidermidis & 6 & $i$ & 15 & 47 \\
\hline Escherichia coli & 7 & 0 & 0 & 0 \\
\hline Escherichia coli + Klebsiella $\mathrm{sp}$ & 1 & 0 & 0 & 0 \\
\hline Escherichia coli + Clostridium perfringens & 1 & 0 & 0 & 0 \\
\hline Enterobacter spp & 2 & 0 & 0 & 0 \\
\hline Salmonella typhi & 2 & 0 & 0 & 0 \\
\hline Proteus mirabilis & 1 & 0 & 0 & 0 \\
\hline Klebsiella spp & 0 & 1 & 0 & 0 \\
\hline Acinetobacter sp & 0 & 1 & 0 & 0 \\
\hline Aeromonas sp & 1 & 0 & 0 & 0 \\
\hline Pseudomonas sp & 1 & 0 & 0 & 0 \\
\hline Haemophilus influenzae & 1 & 0 & 0 & 0 \\
\hline Coryneform organism & $i$ & 0 & 3 & 17 \\
\hline Bacillus sp & 0 & 0 & 1 & 1 \\
\hline Non-fermenting coliform & 0 & 0 & $i$ & 1 \\
\hline Bacteroides sp & 0 & 1 & 0 & 0 \\
\hline Aspergillus spp & 0 & 0 & 2 & 0 \\
\hline Total & 51 & 6 & $2 \overline{6}$ & 66 \\
\hline
\end{tabular}

DETECTION OF POSITIVE CULTURES

Of 270 positive Malthus cultures of clinical importance, $51(19 \%)$ were not detected by the Malthus automatic detection routine (table 2). Similarly, of 114 Malthus cultures containing contaminants, $26(23 \%)$ were not detected by the automatic detection routine (table 2). Causes of failure of the automatic detection routine for the 77 cultures are given in table 3 .

Six $(2 \%)$ of the 273 positive conventional cultures of clinical importance and $66(15 \%)$ of the 451 conventional cultures from which contaminants were isolated were not detected before terminal subculture (table 2).

False positive signals with the Malthus system were

Table 3 Cause of failure of Malthus automatic detection routine to recognise organisms in 77 cultures

\begin{tabular}{lc}
\hline Cause of failure & $\begin{array}{l}\text { No of } \\
\text { cultures }\end{array}$ \\
\hline $\begin{array}{l}\text { Conductivity curve not recognised by detection } \\
\text { routine }\end{array}$ & 38 \\
$\begin{array}{l}\text { Electrode failure } \\
\text { Machine/operator failure leading to loss of data }\end{array}$ & 4 \\
$\begin{array}{l}\text { Cultures incubated overnight before being attached } \\
\text { to the machine and no subsequent conductivity } \\
\text { changes indicative of the presence of micro-organ- }\end{array}$ & 7 \\
$\begin{array}{l}\text { isms } \\
\text { Conductivity changes indicative of the presence of } \\
\text { micro-organisms were in progress when the culture }\end{array}$ & \\
$\begin{array}{l}\text { was attached to the machine and were not recogn- } \\
\text { ised by the detection routine }\end{array}$ & 7 \\
$\begin{array}{l}\text { Cultures promptly attached to the machine but no } \\
\text { conductivity changes indicative of growth }\end{array}$ & 7 \\
\begin{tabular}{l} 
Total \\
\hline
\end{tabular} & 77 \\
\hline
\end{tabular}

noted in $438(13.0 \%)$ cultures, and with the conventional system in $67(2.0 \%)$ cultures.

\section{TIME TO DETECTION OF POSITIVE CULTURES}

Comparison was made of the relative detection times by the Malthus and conventional blood culture methods with cultures in which the same organism was detected in both systems, and for which data were available (table 4). Results were profoundly influenced by failure of the automatic detection routine to recognise some conductivity curves. Apart from detection failures in 32 of 49 positive cultures detected more than 24 hours earlier by the conventional system, there was little difference in the detection times between both systems, when the Malthus system was assessed on the basis of consequent actions by laboratory staff between 09.00 hours and 17.30 hours alone. If the Malthus system was assessed on the basis of consequent actions by laboratory staff at the time the apparatus detected growth, and positive results detected by visual inspection of curves in the Malthus system were included, only $30(15 \%)$ positive cultures would have been detected more quickly by the conventional system compared with $94(47 \%)$ by the Malthus system.

Organisms detected more quickly by the Malthus system were of various species. Those detected sooner by the conventional system were largely Gram positive cocci.

USE OF MALTHUS ALARM

The automatic dialling telephone was used to 
Table 4 Relative detection times of positive cultures by Malthus and conventional blood culture methods

\begin{tabular}{lll}
\hline & \multicolumn{2}{l}{$\begin{array}{l}\text { No of cultures if action in response to Malthus } \\
\text { signals was }\end{array}$} \\
\cline { 2 - 3 } Relative detection time $(h)$ & $09.00-17.30 \mathrm{~h}$ & At detection time \\
\hline No difference $(<4)$ & 114 & 36 \\
Malthus sooner by: $4 \cdot 5-8$ & 2 & 33 \\
$8 \cdot 5-24$ & 7 & 22 \\
Total sooner by Malthus & 24 & 94 \\
Conventional sooner by $4 \cdot 5-8$ & 29 & 8 \\
$8 \cdot 5-24$ & 6 & $55\left(16^{*}\right)$ \\
Total sooner by conventional & 13 & 69 \\
\hline
\end{tabular}

*No of positive cultures detected $>24$ hours earlier by the conventional system when Malthus data were examined visually.

interrogate the system at $22.00 \mathrm{~h}$ each evening for a period of 99 days. The system called a member of staff on 50 days, and 65 cultures which triggered the detection routine were examined. Fifteen positive and 11 contaminated cultures were detected. Of these, 11 positive and six contaminated cultures had not been previously detected. The remaining 39 cultures were falsely positive.

\section{Discussion}

It is generally assumed that early detection and susceptibility testing of organisms in blood cultures are desirable to ensure that effective chemotherapy is put into practice. Studies with both conventional ${ }^{4}$ and Bactec $^{5}$ systems have shown that early examination of cultures results in earlier detection of bacteraemia. The Malthus system automatically examines the cultures every 30 minutes and analyses the accumulated data after each examination for evidence of microbial growth. The Malthus system therefore has considerable potential for early detection of positive blood cultures. The potential was not realised in this trial because the automatic detection routine was inadequate.

The Malthus system depends on organisms in blood cultures producing recognisable changes in conductivity. Most organisms gave clear signals, but with some staphylococci changes in conductivity accelerated too slowly to trigger the detection routine. With some streptococci, including pneumococci, changes were very small, and with Aspergillus contaminants, changes were almost unrecognisable. The use of alternative or modified culture media in the Malthus system will probably be required for reliably detecting some of these organisms.

Increasing the sensitivity of the detection routine so as to detect organisms giving weak or slowly accelerating curves is not a problem but ensuring that the increased sensitivity does not result in unacceptably high false positive rates will probably require a more sophisticated detection routine than that currently available. Even with clear conductivity curves and a sensitive detection routine, positive cultures will be missed if the activity giving rise to changes in conductivity is completed before bottles are attached to the system. Hence, as in the previous trial, ${ }^{\prime}$ it proved necessary to subculture those bottles not promptly attached to the apparatus. Seven positive cultures would have been missed if such subcultures had not been done. Only $16 \%$ cultures were taken at night in the current trial from a general hospital population compared with $28 \%$ in the previous trial, ${ }^{1}$ from immunocompromised patients. The difference reflects the lesser urgency of diagnosis in a more general population of patients.

The Malthus system is currently the only blood culture system by which cultures are continuously monitored and positive results automatically signalled. In terms of early detection the main advantage of continuous monitoring may be derived from immediate response to positive signals at any time of the day or night. If this had been the case in the current trial $47 \%$ cultures would have been detected more than four hours earlier by the Malthus system and $32 \%$ more than four hours earlier by the conventional system. Again, failure of the detection routine limited the advantage of the Malthus system. With a more effective detection routine, two thirds of the cultures detected earlier by the conventional system would have been detected at a similar time by both systems, with the result that only $15 \%$ cultures would have been detected earlier by the conventional system.

Unless the laboratory is permanently staffed, immediate response to Malthus signals at any time of the day or night is unlikely to be acceptable on the grounds of practicability and cost. Cultures signalled as positive by the Malthus system during the evening, however, could be examined in a single batch late in the evening. Any positive cultures so detected might be subcultured so that pure cultures and results of direct susceptibility tests would be available the following morning. When the automatic dialling telephone linked to Malthus was used at $22.00 \mathrm{~h}$ each evening to 
interrogate the system for information on whether a positive culture has been detected, useful information was obtained but there was a high rate of false positive signals. Two thirds of the false positive results were due to signals produced by erythrocytes during the first few hours of incubation. The concentration of saponin used in this trial seems to be inadequate for suppression of the "blood effects" in all samples. An alternative agent now in use, $0.5 \% \mathrm{w} / \mathrm{v}$ dextran, ${ }^{6}$ has virtually eliminated false positive signals caused by "blood effects".

Although there were no overall differences in isolation rates between the two systems, strains of $S$ aureus were grown significantly more often from the conventional bottle. The saponin present in the Malthus bottle is unlikely to have been as inhibitory to staphylococci as to some other organisms, notably Streptococcus pneumoniae, which are considerably more sensitive to saponin than staphylococci (unpublished observations).

The conventional bottles were vented in an atmosphere containing carbon dioxide; although carbon dioxide is beneficial for the growth of many organisms, its effect on isolation rates in respect of blood cultures has not to our knowledge been established. Malthus bottles were not vented, but they had a head space of $40 \mathrm{ml}$ air before inoculation. Hence the atmosphere in the Malthus bottle was more equivalent to that produced by transient venting, which has been shown to be as effective as chronic venting. ${ }^{8}$ Indeed, in this and a previous trial' the absence of venting did not suppress Pseudomonas spp. Strains of $S$ aureus have not generally been isolated more often from vented bottles. $^{7-9}$ Although the differences between the Malthus and conventional systems in isolation rates of staphylococci are not explicable in terms of the effects of the atmosphere for incubation noted by others, the small differences between the head space atmospheres of the Malthus and conventional bottles seem to provide the most likely explanation. The lower contamination rate in the Malthus system is probably related to the lower incidence of subculture of the Malthus bottles, as occurred in the previous trial.'

We conclude that the apparatus in its current state of development is unacceptable for routine screening of blood cultures. Limitations of the detection routine and logistical problems of promptly attaching bottles to the machine must be overcome, and false positive rates reduced. If such limitations are overcome, however, there would undoubtedly be savings in labour and materials compared with those used in screening by conventional methods, and clinical benefits might be derived from earlier detection of positive cultures.

We are grateful to Mr D A Kennedy for administrative assistance. This work was supported by a grant from the Department of Health and Social Security.

\section{References}

1 Brown DFJ, Warner M, Taylor CED, Warren RE. Automated detection of micro-organisms in blood cultures by means of the Malthus microbiological growth analyser. J Clin Pathol 1984;37:65-9.

2 Cowan ST, Steele KJ. Manual for the identification of medical bacteria. 2nd ed. London: Cambridge University Press, 1974.

3 Armitage P. Statistical methods in medical research. Oxford: Blackwell Scientific Publications, 1971:127-8.

4 Todd JK, Roe MH. Rapid detection of bacteremia by an early subculture technic. Am J Clin Pathol 1975;64:694-9.

5 Brooks K, Sodeman T. Rapid detection of bacteremia by a radiometric system. Am J Clin Pathol 1974;61:859-66.

6 Curtis GDW, Thomas CD, Johnston HH. A note on the use of dextran in blood cultures monitored by conductance methods. $J$ Appl Bacteriol 1985;58:571-5.

7 Blazevic DJ, Stemper JE, Matsen JM. Effect of aerobic and anaerobic atmospheres on isolation of organisms from blood cultures. J Clin Microbiol 1975;1:154-6.

8 Washington JA. Conventional approaches to blood culture. In: Washington JA, ed. The detection of septicemia. West Palm Beach, Florida: CRC Press Inc, 1978:41-87.

9 Tenney JH, Reller LB, Mirrett S, Weinstein MP, Wang WL. Controlled evaluation of the effect of atmosphere of incubation on detection of bacteremia and fungemia in supplemented peptone broth. J Clin Microbiol 1982;16:437-42.

Requests for reprints to: Dr D F J Brown, Principal Microbiologist, Public Health Laboratory, Level 6, Addenbrooke's Hospital, Hills Road, Cambridge CB2 2QW, England. 